Ann. Biol. anim. Bioch. Biophys., I963, 3 (3), 243-253

\title{
INFLUENCE DE LA NATURE DU RÉGIME ALIMENTAIRE SUR L'EXCRÉTION FÉCALE DE PHOSPHORE ENDOGĖNE CHEZ LE VEAU
}

\author{
L. GUEGUEN \\ avec la collaboration technique de Marguerite-Marie Wambergue et de R. Tromas \\ Laboratoire des Métabolismes, \\ Centre national de Recherches zootechniques, Jouy-en-Josas (Seine-et-Oise)
}

SOMMAIRE

La proportion de phosphore endogène dans le phosphore fécal total a été déterminée chez 6 veaux âgés de 7.8 semaines et soumis à deux régimes alimentaires différents $: 3$ veaux n'ont reçu que du lait entier (régime $L$ ) et 3 veaux ont suivi un régime de sevrage précoce comprenant une quantité limitée de lait, un aliment concentré sec et du foin de graminées (régime LCF).

Dans chacun des lots, 2 veaux ont servi à l'estimation de l'excrétion fécale de $\mathrm{P}$ endogène à l'aide d'une méthode de dilution isotopique après injection de ${ }^{32} \mathrm{P}$, et un veau a été utilisé pour l'étude des variations des excrétions fécales et urinaires d'une dose ingérée de radioisotope.

La proportion de $\mathrm{P}$ endogène dans le $\mathrm{P}$ fécal total était de $5 \mathrm{I} \mathrm{p}$. I oo chez les veaux du régime $\mathrm{L}$ et de $45 \mathrm{p}$. Ioo chez les veaux du régime LCF ; mais, comme l'excrétion fécale totale de $\mathrm{P}$ était beaucoup plus importante chez les veaux recevant de l'aliment concentré et du foin, il en est résulté des pertes fécales endogènes journalières de $P$ de $0,15 \mathrm{~g}$ pour le régime $\mathrm{L}$ et de $\mathrm{r}, 9 \mathrm{~g}$ pour le régime $\mathrm{LCF}$, soit $\mathrm{I} 3$ fois plus. Plusieurs hypothèses ont été émises pour expliquer ces différences entre régimes alimentaires et pour discuter le déterminisme de l'excrétion fécale endogène.

Le coefficient d'utilisation digestive réelle de $\mathbf{P}$ était de $98 \mathrm{p}$. 100 pour le lait et de $80 \mathrm{p}$. 100 pour les aliments secs. La nature physique du lait, qui ne favorise pas les pertes métaboliques d'éléments minéraux dans les fèces, semble être un facteur important de la très bonne utilisation digestive apparente de $\mathbf{P}$ du lait (97 p. I0o).

L'étude des courbes représentant l'évolution des excrétions cumulées de ${ }^{32} \mathrm{P}$ dans l'urine et les fèces, après ingestion d'un repas marqué, est particulièrement intéressante. Ainsi, au bout de 8 jours, $60 \mathrm{p}$. roo de la dose de ${ }^{32} \mathrm{P}$ ingérée a été éliminée dans les excreta par les veaux du régime LCF, contre I4 p. roo seulement chez les veaux n'ayant reçu que du lait. En outre, la durée du transit dans le tube digestif de la totalité du $\mathrm{P}$ d'un repas de lait a pu être estimée à go heures environ.

Ces expériences montrent notamment que le besoin d'entretien en $\mathrm{P}$ peut être très élevé chez les jeunes animaux et que ce besoin est susceptible de varier considérablement suivant la nature du régime alimentaire.

La nature du régime alimentaire influe considérablement sur le degré d'utilisation des éléments minéraux par l'animal. Dans une étude récente (GuEguen et 
MATHIEU, I962), nous avions pu mettre en évidence quelques différences fondamentales dans le métabolisme des éléments minéraux, d'une part chez des veaux ne recevant que du lait, d'autre part chez des veaux sevrés tôt et recevant un aliment concentré et du foin.

Afin d'expliquer certaines différences dans 1'utilisation digestive du calcium et du phosphore en particulier, nous avions émis l'hypothèse d'une plus forte excrétion endogène fécale de ces éléments chez les veaux recevant des aliments solides. Cette hypothèse avait également été formulée par SмIтH (I96I) pour expliquer la diminution de l'utilisation digestive apparente du calcium et du magnésium chez des veaux recevant exclusivement du lait mais pouvant mâcher des copeaux de bois. En effet, cette mastication entraînerait une sécrétion accrue de sucs digestifs et en particulier de salive, contribuant ainsi à augmenter l'excrétion endogène de certains éléments minéraux.

Nous avons voulu vérifier cette hypothèse dans le cas du phosphore, chez des veaux âgés de 6 à 8 semaines. Pour cela, nous avons estimé 1'excrétion fécale de phosphore endogène au moyen d'une méthode de dilution isotopique simplifiée (KILIBER et al., I95 I ; LOFGREEN et KLEIBER, I954) que nous avons décrite antérieurement (GUEGUEN, I962), d'une part chez des veaux recevant exclusivement du lait (régime L), d'autre part chez des veaux sevrés tôt (6 semaines) et recevant dès l'âge de $x$ semaine un aliment concentré sec, du foin à volonté et de l'eau (régime $\mathrm{L}, \mathrm{CF})$.

Dans le but de suivre l'évolution comparative des éliminations fécale et urinaire de ${ }^{32} \mathrm{P}$, nous avons complété ce travail par un essai comportant l'ingestion d'une dose unique de radioisotope par un animal de chacun des deux lots.

\section{MATÉRIEL EXPÉRIMENTAL, ET MÉTHODES UTILISÉES}

\section{$\left.\mathrm{I}^{\circ}\right)$ Animaux et alimentation}

Six veaux mâles de race Normande ont été répartis, à la fin de leur première semaine, en deux lots : 3 veaux ont reçu le régime L (lait seul) et 3 veaux ont reçu le régime LCF (lait, concentré sec et foin).

Le lait de mélange a été offert deux fois par jour, à la température de $35^{\circ} \mathrm{C}$, suivant la répartition indiquée dans le tableau $\mathbf{r}$.

TABLEAU I

Quantités de lait distribuées, en litres par jour

\begin{tabular}{|c|c|c|}
\hline Age, en semaines & Régime L & Régime LCF \\
\hline 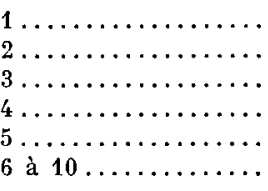 & $\begin{array}{l}4 \text { à } 6 \\
6 \\
7 \\
8 \\
8 \\
8\end{array}$ & $\begin{array}{l}4 \\
5 \\
6 \\
4 \\
2 \\
0\end{array}$ \\
\hline
\end{tabular}


Dans le régime $\mathrm{I}$, les veaux n'ont consommé que du lait.

Dans le régime LCF, l'aliment concentré, le foin et l'eau ont été offerts dès la deuxième semaine. Le foin a été distribué à volonté tandis que la consommation d'aliment concentré a été limitée à I $000 \mathrm{~g}$ par jour. Ainsi, il a été possible, pour chaque régime, de maintenir des apports alimentaires approximativement constants durant toute la durée des expériences : 8 litres de lait entier par jour pour les veaux du régime $\mathrm{L}, 400 \mathrm{à} 500 \mathrm{~g}$ de foin et 800 à $900 \mathrm{~g}$ d'aliment concentré pour les veaux du régime LCF à partir de leur $7^{\mathbf{e}}$ semaine.

Les compositions de l'aliment concentré et du composé minéral sont indiquées dans le tableau 2.

TABLEAU 2

Compositions de l'aliment concentré et du composé minéral utilisé

\begin{tabular}{|c|c|c|c|}
\hline \multicolumn{2}{|l|}{ Aliment concentré, p. 100} & \multicolumn{2}{|l|}{ Composé minéral, p. 100} \\
\hline 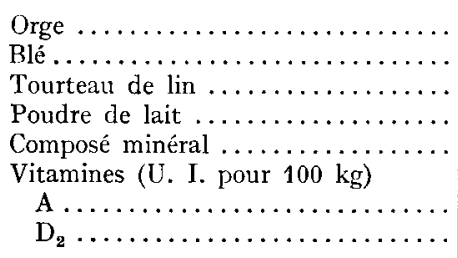 & $\begin{array}{r}30 \\
27 \\
30 \\
10 \\
3 \\
1200000 \\
400000\end{array}$ & $\begin{array}{l}\text { Phosphate bicalcique. } \ldots \ldots \ldots \ldots \ldots \\
\text { Carbonate de calcium } \ldots \ldots \ldots \ldots \ldots \\
\text { Chlorure de sodium } \ldots \ldots \ldots \ldots \ldots \ldots \\
\text { Magnésie } \ldots \ldots \ldots \ldots \ldots \ldots \ldots \ldots \\
\text { Sulfate ferreux } \ldots \ldots \ldots \ldots \ldots \ldots \\
\text { Sulfate de cuivre } \ldots \ldots \ldots \ldots \ldots\end{array}$ & $\begin{array}{l}40 \\
36 \\
18 \\
5 \\
0,40 \\
0,04\end{array}$ \\
\hline
\end{tabular}

Les teneurs en $\mathrm{Ca}$ et $\mathrm{P}$, en grammes par kilogramme de matière sèche, étaient respectivement de 6,3 et 3,0 pour le foin de graminées et de $x 5,9$ et II, 5 pour l'aliment concentré.

Ainsi, l'apport journalier de $\mathrm{Ca}$ et de $\mathrm{P}$ a été respectivement en moyenne de $10,0 \mathrm{~g}$ et $7,5 \mathrm{~g}$ chez les veaux du régime $\mathrm{L}$ et de $19,7 \mathrm{~g}$ et $9,9 \mathrm{~g}$ chez les veaux du régime $\mathrm{LCF}$.

\section{$\left.2^{\circ}\right)$ Déroulement de l'expérience}

Les veaux ont été placés en cages à métabolismes (permettant de séparer les urines et les fèces) environ 8 jours avant le début de chaque expérience, afin d'obtenir un niveau d'ingestion d'aliments pratiquement constant.

Expérience avec injection de ${ }^{32} \mathrm{P}$. - Deux veaux du régime L, âgés de 6 semaines et pesant en moyenne $65 \mathrm{~kg}$, ont reçu par injection sous-cutanée $4,5 \mathrm{mCi} \mathrm{de}{ }^{32} \mathrm{P}$ sous forme de solution neutre stérile isotonique de $\mathrm{HNa}_{2} \mathrm{PO}_{4}$, tandis que 2 veaux du régime LCF âgés de 7 semaines et pesant en moyenne $68 \mathrm{~kg}$, ont reçu, également par injection sous-cutanée, environ $6 \mathrm{mCi}$ de ${ }^{32} \mathrm{P}\left({ }^{1}\right)$.

Des échantillons représentatifs d'urine et de fèces ont été prélevés chaque jour jusqu'au $2^{\circ}$ jour après l'injection et des prises de sang ont été effectuées périodiquement dans l'une des veines jugulaires, du $3^{\mathrm{e}}$ au $20^{\mathrm{e}}$ jour.

Afin d'éviter les contaminations mutuelles entre les prélèvements successifs de fèces, contamina. tions risquant d'être importantes au cours des premiers jours suivant l'injection, les bacs à fèces étaient recouverts de feuilles en matière plastique enlevées quotidiennement avec leur contenu.

Expérience avec ingestion de ${ }^{32} \mathrm{P}$. - Une dose unique de I $_{7} 7 \mu \mathrm{Ci}$ de ${ }^{22} \mathrm{P}$, mélangée à 4 litres de lait, a été administrée à un veau du régime $L$, âgé de 9 semaines et pesant $85 \mathrm{~kg}$, tandis que le veau correspondant du régime LCF, âgé de ro semaines et pesant $80 \mathrm{~kg}$ recevait par sonde œsophagienne $\mathrm{I} 47 \mu \mathrm{Ci}$ de ${ }^{32} \mathrm{P}$.

Les fèces et l'urine de ces deux veaux ont été recueillis quantitativement et séparément chaque jour (ou plusieurs fois par jour) durant 8 à Io jours après l'ingestion du repas marqué ; après pesée et homogénéisation, des prélèvements représentatifs ont été effectués.

(1) En prévision d'une élimination plus rapide, chez les veaux du régime $\mathbf{L C F}$, de la radioactivité injectée ou ingérée, nous avons administré à ces veaux des doses plus fortes de ${ }^{32} \mathbf{P}$ afin d'assurer des comptages valables durant les derniers jours de l'expérience. 


\section{$\left.3^{0}\right)$ Préparation des échantillons et méthodes de dosage}

Le sang, recueilli sur héparine, a été centrifugé immédiatement pour séparer le plasma.

Pour le plasma et l'urine, les mesures de radioactivité ont été effectuées directement sur des prises de $\mathrm{I}$ ou $2 \mathrm{ml}$ et les dosages de $\mathrm{P}$ total après minéralisation nitro-perchlorique d'une prise de ro $\mathrm{ml}$.

Dans le cas des fèces, les mesures ont porté sur la solution obtenue après dissolution du résidu de calcination à $530-55^{\circ} \mathrm{C}$ d'une prise de $20 \mathrm{~g}$ environ de fèces frais, préalablement séchée sur un bain de sable.

Les dosages de $\mathrm{P}$ total ont été effectués par colorimétrie du phosphovanadomolybdate d'ammonium et les comptages de radioactivité à l'aide d'un compteur Geiger-Muller à fenêtre mince $\left(2 \mathrm{mg} / \mathrm{cm}^{2}\right)$.

\section{RÉSULTATS}

$\left.\mathrm{I}^{\circ}\right)$ Evolution des radioactivités spécifiques du phosphore du plasma et des fèces après injection de ${ }^{32} \mathrm{P}$

Les figures I et 2 montrent que, au bout de quelques jours, ces évolutions sont très régulières et indiquent, en accord avec LoFGREEN et KLEIBER (I954), une
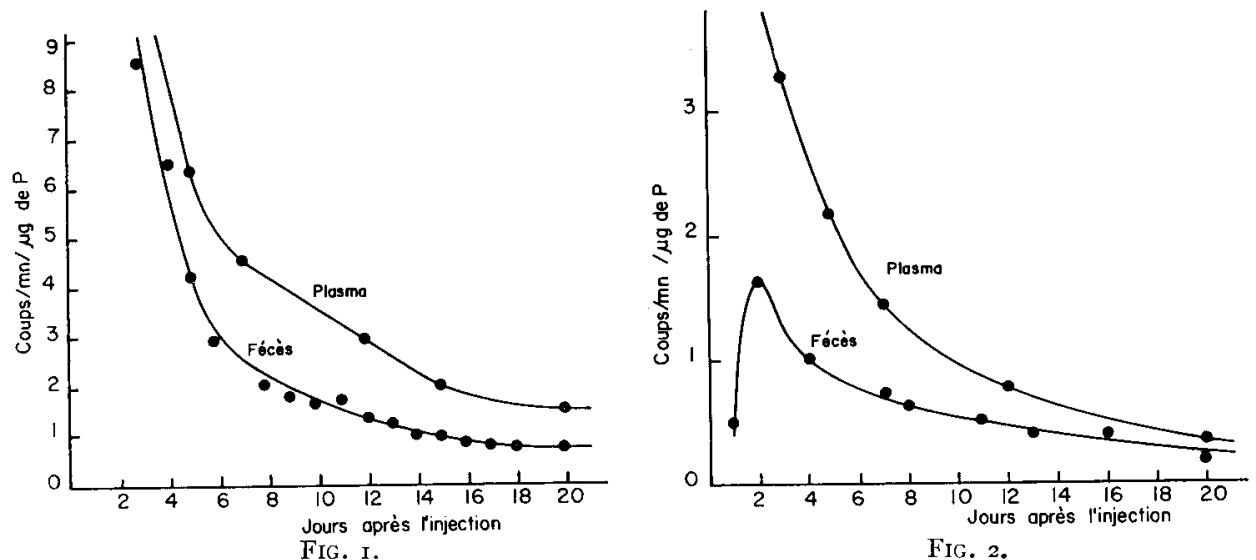

FIG. I. - Évolution des radioactivités spécifiques moyennes de $P$ du plasma et des fèces chez des veaux recevant du foin et un aliment concentré, après injection de ${ }^{32} \mathrm{P}$

FIG. 2. - Évolution des radioactivités spécifiques moyennes de $P$ du plasma et des fèces chez des veaux nourris au lait, apres injection de ${ }^{32} P$

période de décroissance pratiquement linéaire, allant $\mathrm{du} 7^{\mathrm{e}}$ au $\mathrm{I} 3^{\mathrm{e}}$ jour après l'injection pour le plasma et du $8^{\mathrm{e}}$ aur $\mathrm{I}_{4}^{\mathrm{e}}$ jour pour les fèces.

L'étude de l'évolution de la radioactivité spécifique de $\mathrm{P}$ des fèces durant les premiers jours a montré des décalages de 2 jours entre plasma et fèces pour les veaux du régime $\mathrm{L}$ (lait seul) et de I jour et demi pour les veaux du régime $\mathrm{LCF}$ (lait, concentré, foin).

La radioactivité spécifique de $\mathrm{P}$ du plasma est beaucoup plus élevée chez les veaux ne recevant pas de lait : ceci résulte de la dose plus forte de ${ }^{32} \mathrm{P}$ injectée à ces derniers veaux et aussi de la plus grande teneur en $\mathbf{P}$ total du plasma des veaux recevant du lait ( $445 \mathrm{ppm}$ en moyenne contre I05 ppm chez les veaux $\mathrm{LCF}$ ).

Nous avons également vérifié que, dans le plasma, le phosphore total avait la même radioactivité spécifique que le phosphore acido-soluble. 


\section{$\left.2^{\circ}\right)$ Excrétion endogène fécale et utilisation digestive réelle du phosphore}

En utilisant les parties linéaires des courbes de décroissance des radioactivités spécifiques de $\mathrm{P}$ du plasma et des fèces (fig. I et 2 ) et en corrigeant les activités de $\mathrm{P}$ des fèces pour tenir compte du décalage avec le plasma, on obtient, en faisant le rapport des activités spécifiques moyennes de $\mathrm{P}$ des fèces et du plasma, la proportion de $\mathrm{P}$ endogène dans le $\mathrm{P}$ fécal total (tabl. 3).

\section{TABLEAU 3}

Radioactivités spécifiques moyennes du phosphore du plasma et des fèces durant la période d'évolution linéaire (en coups/mn/mg de P)

\begin{tabular}{|c|c|c|c|c|c|}
\hline & & \multicolumn{2}{|c|}{ Veaux L } & \multicolumn{2}{|c|}{ Veaux LCF } \\
\hline Plasma & $\begin{array}{l}\text { Jour } \ldots \ldots \ldots \ldots \ldots \ldots \ldots \\
\text { Activitê } \ldots \ldots \ldots \ldots \ldots \ldots\end{array}$ & $\begin{array}{c}7^{e} \\
1440\end{array}$ & $\begin{array}{c}12^{\mathrm{e}} \\
780\end{array}$ & $\begin{array}{c}8^{0} \\
4300\end{array}$ & $\begin{array}{r}13^{e} \\
2550\end{array}$ \\
\hline Fèces & Jour $\ldots \ldots \ldots \ldots \ldots \ldots \ldots$ & $\begin{array}{r}9^{\mathrm{e}} \\
660\end{array}$ & $\begin{array}{l}14^{\circ} \\
440\end{array}$ & $\begin{array}{c}9^{e} \\
2100\end{array}$ & $\begin{array}{r}14^{\mathrm{e}} \\
1050\end{array}$ \\
\hline Fèces/Plasma & $\ldots \ldots \ldots \ldots \ldots \ldots \ldots \ldots$ & 0,46 & 0,56 & 0,49 & 0,41 \\
\hline
\end{tabular}

TABLEAU 4

Bilan du phosphore

Age, en semaines $\ldots \ldots \ldots \ldots \ldots \ldots \ldots \ldots \ldots$

Poids vif moyen, $\mathrm{kg}$.

Ingéré, g par jour

Fécal, g par jour $\ldots \ldots \ldots \ldots \ldots \ldots \ldots \ldots \ldots \ldots \ldots$

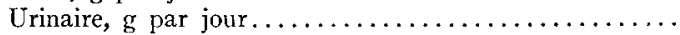

Urinaire

Fécal + Urinaire

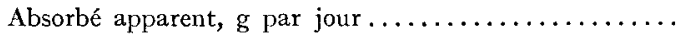

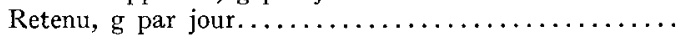

C. U. D. (1) apparent, p. $100 \ldots \ldots \ldots \ldots \ldots \ldots \ldots$

$P$ fécal endogène

$\mathbf{P}$ fécal total $\times 100$

Endogène fécal, g par jour

Exogène fécal, $g$ par jour

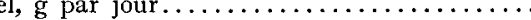

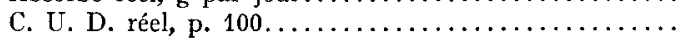

\begin{tabular}{|c|c}
\hline Régime L & Régime ICF \\
\hline 7,8 & $8-9$ \\
75 & 75 \\
& \\
\hline 10,5 & 11,2 \\
0,3 & 4,2 \\
1,8 & 2,4 \\
85,7 & 36,3 \\
10,2 & 7,0 \\
8,4 & 4,6 \\
97,0 & 63,0 \\
& 45,0 \\
51,0 & 1,9 \\
0,15 & 8,3 \\
0,15 & 79,6 \\
10,3 & \\
98,5 & \\
&
\end{tabular}

(1) Coefficient d'utilisation digestive. 
Ces rapports moyens sont de $0,5 \mathrm{I}$ chez les veaux du régime $L_{1}$ et de 0,45 chez les veaux du régime $\mathrm{LCF}$. Ainsi, respectivement, $5 \mathrm{I}$ et $45 \mathrm{p}$. Ioo du $\mathrm{P}$ fécal est endogène, la différence entre les deux régimes n'étant pas significative.

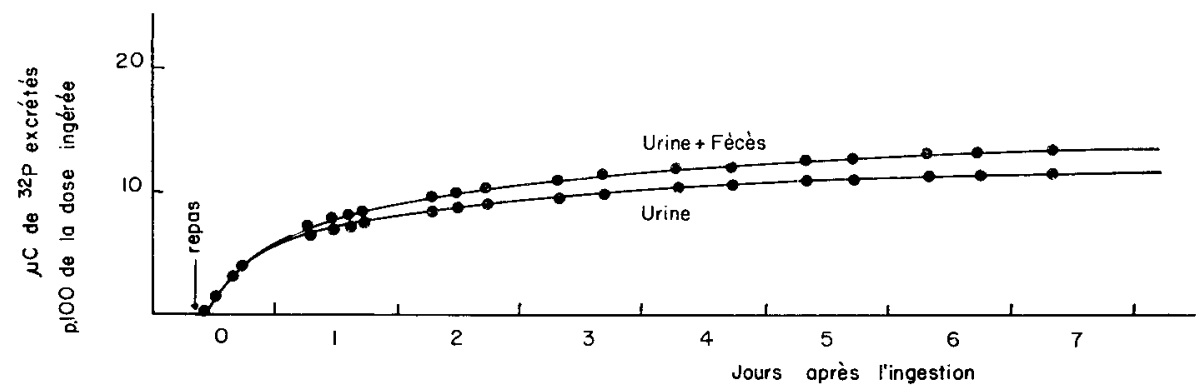

FIG. 3. - Éliminations urinaire et fécale cumulées de ${ }^{32} \mathrm{P}$ ingéré, par un veau nourri au lait

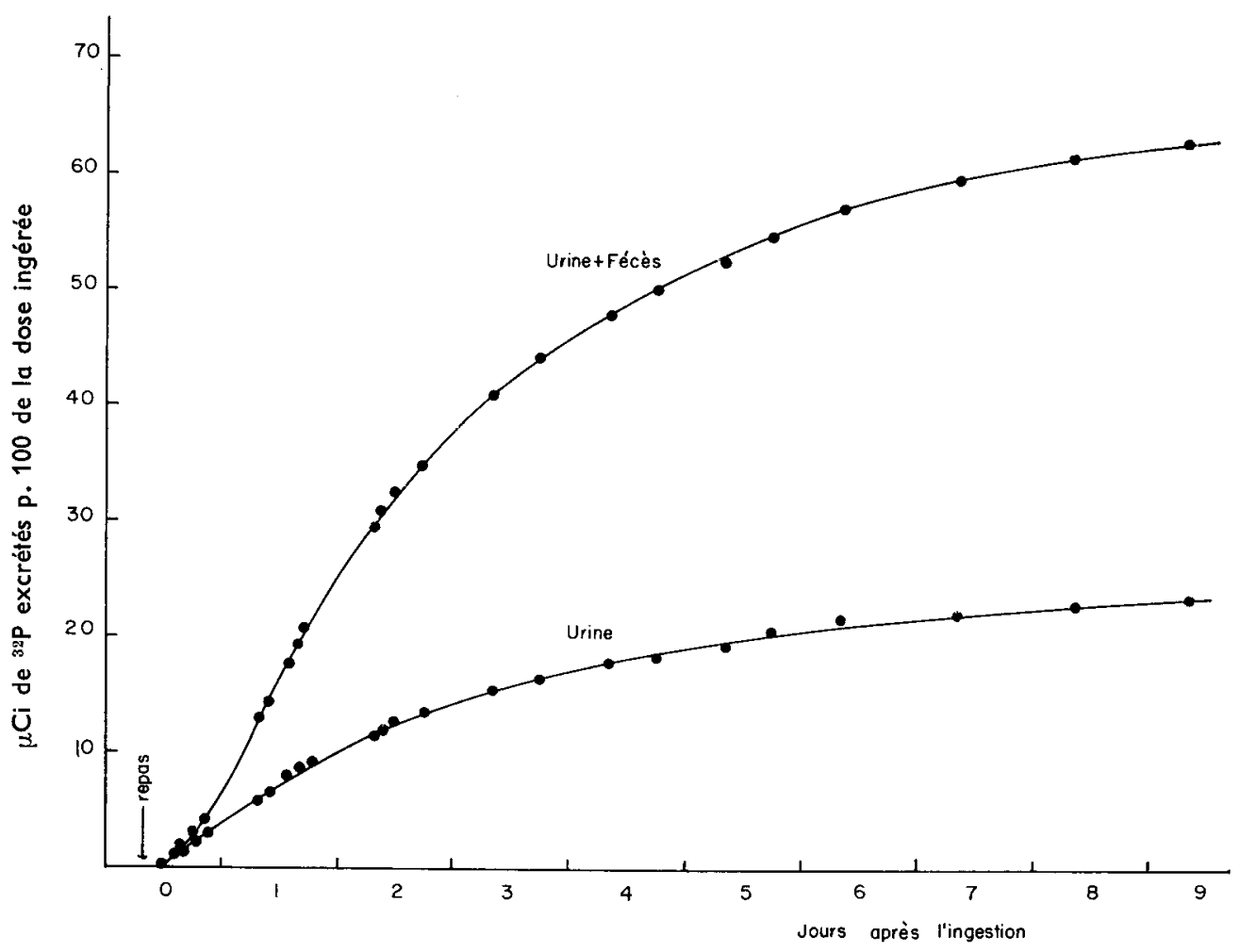

FIG. 4. - Éliminations urinaire et fécale cumulées de ${ }^{32} P$ ingéré, par un veau sevré

Nous avons utilisé dans le tableau 4 les résultats de bilans obtenus lors d'une expérience antérieure (GUEGUEN et MATHIEU, I962) ; en effet, ces résultats proviennent de bilans de longue durée effectués sur un plus grand nombre d'animaux, et sont donc plus significatifs que les résultats de bilans que nous avons obtenus au cours de l'essai présent. Les régimes alimentaires utilisés dans ces deux expériences successives étaient très comparables et nous avons donc admis que les proportions de 
$P$ fécal endogène dans le $P$ fécal total trouvées dans l'expérience présente pouvaient être appliquées à ces résultats de bilans.

Ainsi, la perte métabolique fécale de $\mathrm{P}$ est en moyenne $\mathrm{I}_{3}$ fois plus forte chez les veaux du régime LCF et, compte tenu des différences d'utilisation digestive réelle de $\mathrm{P}$ des deux rations, 1'apport alimentaire de $\mathrm{P}$ nécessaire pour couvrir cette perte fécale est I6 fois plus élevé dans le cas des veaux sevrés.

Le coefficient d'utilisation digestive réelle du phosphore des aliments secs est de 80 p. I0o, contre 98 p. Ioo pour le phosphore du lait.

\section{$\left.3^{\circ}\right)$ Éliminations urinaire et fécale de ${ }^{32} \mathrm{P}$ après ingestion d'un repas marqué}

L'élimination cumulée de ${ }^{32} \mathrm{P}$ dans 1'urine et dans la totalité des excreta, après l'ingestion d'un repas marqué, est représentée sur les figures 3 et 4 . Ainsi, en 8 jours, les veaux au lait n'ont éliminé que I4 p. roo de la dose ingérée, tandis que les veaux du régime LCF ont éliminé dans leurs excreta environ $60 \mathrm{p}$. Ioo de la dose.

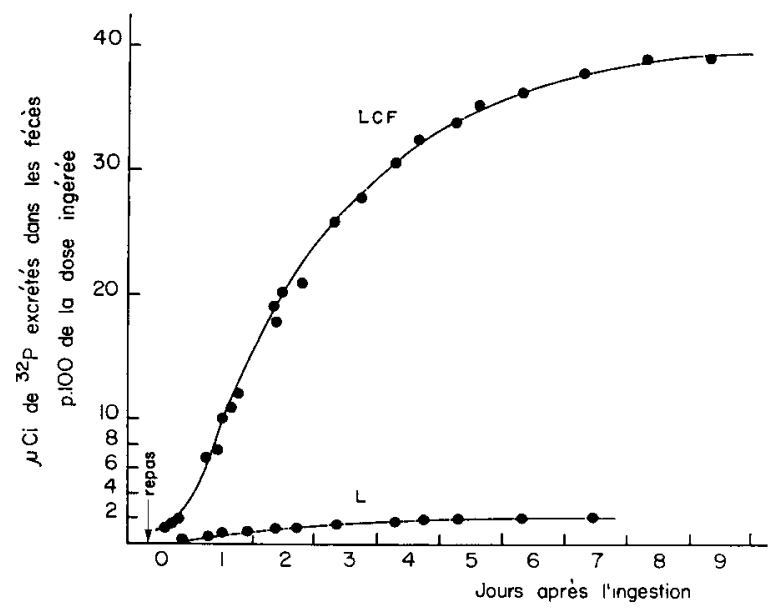

FIG. 5. - Variations de l'excrétion fécale cumulée de ${ }^{32} P$ ingéré par deux veaux recevant des régimes alimentaires différents

La représentation de 1'élimination fécale cumulée d'une dose ingérée de ${ }^{32} \mathrm{P}$ (fig. 5) montre que le métabolisme intestinal de $\mathrm{P}$ est très différent chez les deux lots de veaux. Ainsi, au bout de 8 jours, près de $40 \mathrm{p}$. Ioo de la dose ingérée a été excrété dans les fèces chez les veaux LCF et moins de 2 p. Ioo seulement chez les veaux $L_{\text {. }}$.

La comparaison des pentes des parties linéaires des courbes figurant les excrétions fécales cumulées, à partir de quelques jours après l'ingestion du repas marqué (fin de l'excrétion du ${ }^{32} \mathrm{P}$ exogène) indique approximativement le rapport des excrétions fécales de $\mathrm{P}$ endogène. Ainsi, nous avons ici un rapport des pentes de l'ordre de $I 8$, ce qui confirme les résultats obtenus par la méthode de dilution isotopique.

\section{$\left.4^{\circ}\right)$ Application à l'estimation de la vitesse du transit intestinal du phosphore alimentaire}

La représentation à une échelle plus grande (fig. 6) de l'évolution de l'excrétion fécale cumulée de $P$ dans le cas des veaux du régime $I_{1}$, permet de définir deux courbes distinctes : le changement de pente correspond à la fin de l'excrétion de ${ }^{32} \mathrm{P}$ 
exogène et la dernière partie de la courbe, de pente très faible, ne représente plus que l'excrétion fécale de ${ }^{32} \mathrm{P}$ endogène.

Ainsi, on peut estimer à go heures environ la durée du transit de la totalité de $P$ ingéré, et à 80 heures environ le temps écoulé entre le début et la fin de 1'excrétion fécale d'un repas de lait.

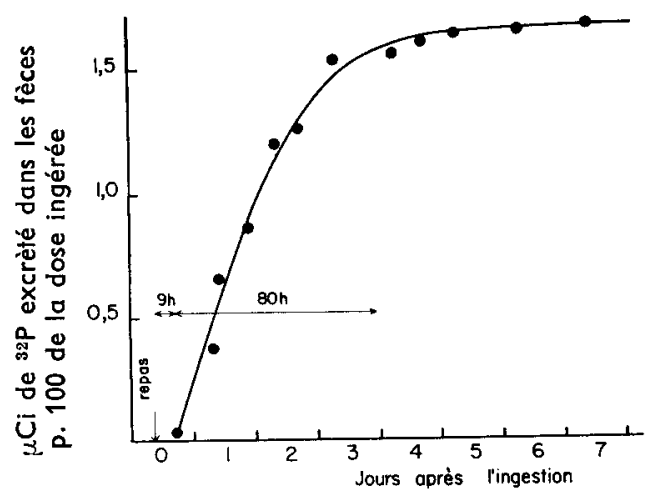

Fig. 6. - Évolution de lélimination fécale cumulée de ${ }^{32} P$ ingéré, chez un veau nourri au lait

Cette technique, déjà utilisée par Hansard et al. (1954), peut difficilement être appliquée aux veaux du régime $\mathrm{LCF}$, où l'excrétion fécale endogène est importante et le changement de pente difficile à définir (fig. 5).

\section{DISCUSSION}

\section{Io) Excrétion fécale de phosphore endogène}

$\mathrm{L}$ a proportion de $\mathrm{P}$ fécal endogène dans le $\mathrm{P}$ fécal total est voisine pour les detux régimes : $5 \mathrm{I}$ p. roo chez les veaux ne recevant que du lait (régime $\mathrm{L}$ ) et $45 \mathrm{p}$. Ioo chez les veaux recevant un aliment concentré et du foin (régime $\mathrm{L}_{\mathrm{CF}}$ ). Cependant, l'excrétion fécale de $\mathrm{P}$ étant très faible chez les veaux ne recevant que du lait, ceci se traduit par une grande différence entre les pertes métaboliques fécales de $\mathrm{P}$ : 0,I5 $\mathrm{g}$ par jour chez les veaux $\mathrm{L}$ et $\mathrm{I}, 9 \mathrm{~g}$ par jour chez les veaux LCF, soit I3 fois plus (tabl. 4).

Il est intéressant de remarquer que l'excrétion fécale de $\mathrm{P}$ endogène semble être en relation étroite avec l'excrétion fécale de matière sèche et non pas avec 1'ingestion de matière sèche. En effet, les quantités de matière sèche excrétées par les fèces chez les veaux des deux régimes sont dans un rapport voisin de ro, tandis que le rapport des quantités de matière sèche ingérées n'est que de 1,5 environ.

Plusieurs hypothèses peuvent être formulées pour expliquer ces différences :

- le volume des sécrétions digestives, et principalement de la salive, est considérablement plus important chez les veaux recevant des aliments solides. Ainsi, la quantité de $\mathrm{P}$ provenant de la salive et non réabsorbé serait accrue ; 
- la desquamation de cellules de la muqueuse intestinale serait favorisée, chez les veaux du régime LCF, par un chyme beaucoup plus volumineux et plus consistant, et peut-être par une évolution dans la structure des parois du tube digestif, liée au passage du monogastrique au ruminant;

- le rumen étant fonctionnel chez les veaux du régime LCF, une certaine quantité de microorganismes, agents de mobilisation du phosphore salivaire, serait excrétée dans les fèces et contribuerait ainsi à l'élimination de $\mathrm{P}$ endogène ;

- 1'excrétion urinaire de $\mathrm{P}$ est faible chez les veaux du régime $\mathrm{LCF}$ et, en outre, l'apport énergétique et azoté ne permet sans doute pas une rétention maximum du phosphore absorbé. En conséquence, le $\mathrm{P}$ fécal endogène pourrait résulter d'une excrétion normale dans le tube digestif (différente de la sécrétion par les sucs digestifs) de l'excès de $P$ circulant. En fait, dans le régime $I, C F$, les quantités de $P$ retenues et excrétées par 1'urine sont beaucoup plus faibles que dans le régime $L$, tandis que les quantités réellement absorbées sont peu différentes (tabl. 4).

Toutefois, si les trois premières hypothèses formulées étaient valables, on pourrait également considérer l'excrétion fécale endogène de $\mathrm{P}$ comme étant le phénomène primaire duquel dépendrait l'importance de l'excrétion urinaire et peutêtre de la rétention. Dans ce cas, l'excrétion fécale endogène de $\mathrm{P}$ serait aussi importante chez des veaux recevant une ration de même nature (permettant de maintenir constants les facteurs susceptibles d'influer sur l'excrétion endogène) mais favorable à une bonne rétention $\mathrm{du}$ phosphore absorbé (ingestions réduites de $\mathrm{P}$ et apports élevés en énergie et en azote). Inversement, si l'excrétion fécale de $\mathrm{P}$ endogène était surtout un phénomène secondaire, elle devrait également être accrue chez des veaux recevant du lait enrichi en $P$.

Ces deux aspects du déterminisme de l'excrétion fécale de $\mathrm{P}$ endogène doivent faire l'objet de prochaines expériences.

\section{$\left.2^{\circ}\right)$ Utilisation digestive réelle $d u$ phosphore}

L'absorption réelle de $\mathrm{P}$ des aliments secs (aliment concentré et foin) est très bonne ( $80 \mathrm{p}$. IOo) mais reste inférieure à l'absorption de $\mathrm{P}$ du lait (98 p. I0o), ce qui peut être attribué à l'utilisation digestive incomplète du phosphore phytique fourni par les céréales. Cependant, à quantités ingérées voisines, les quantités absolues de $\mathrm{P}$ réellement absorbées dans les deux régimes sont peu différentes (Io,3 g et 8,9 $\mathrm{g}$ par jour), la rétention plus forte du $\mathrm{P}$ fourni par le lait résultant principalement du fait que, dans ce cas, l'excrétion endogène fécale est négligeable. Ainsi, la valeur du lait en tant que source de $P$ pour les animaux serait due, non pas seulement à la forme chimique facilement utilisable du phosphore, mais aussi à la nature physique du lait qui ne favorise pas les pertes métaboliques de $\mathrm{P}$ dans les fèces.

\section{$\left.3^{\circ}\right)$ Variations des éliminations urinaire et fécale de ${ }^{32} \mathrm{P}$ ingéré. Vitesse du transit digestif $d u$ phosphore}

L'élimination par les excreta d'une dose ingérée de ${ }^{32} \mathrm{P}$ est beaucoup plus importante chez les veaux recevant le régime LCF (6o p. Ioo de la dose est éliminé en 8 jours, contre I4 p. roo chez les veaux L). Ceci résulte principalement des différences d'élimination par les fèces, le phosphore non absorbé et le phosphore endogène étant beaucoup plus abondants chez les veaux du régime LCF (fig. 3 et 4 ). 
L'étude des courbes d'excrétion fécale cumulée de ${ }^{32} \mathrm{P}$ (fig. 5 et 6 ) permet de séparer approximativement les évolutions des excrétions exogène et endogène de cet élément. Sachant que la partie de la courbe ayant une pente forte correspond à l'élimination massive du ${ }^{32} \mathrm{P}$ exogène, il est possible, d'une part, de situer le début et la fin de cette excrétion du phosphore alimentaire et, d'autre part, d'estimer l'importance relative de l'excrétion endogène (correspondant aux autres parties de la courbe). Contrairement à ce qui se passe chez les veaux ne recevant que du lait, l'excrétion du ${ }^{32} \mathrm{P}$ exogène est précédée chez les veaux LCF d'une courte période d'excrétion de ${ }^{32} \mathrm{P}$ endogène (fig. 5) atteignant les fèces par la voie la plus rapide, le sang.

La fin de l'excrétion fécale de ${ }^{32} \mathrm{P}$ ingéré, correspondant au changement de pente de la courbe, est très nette chez les veaux du régime $L$ (fig. 6 ) et il est donc possible d'estimer à environ 90 heures la durée totale du séjour dans le tube digestif du phosphore d'un même repas. HANSARD et al. (I954), par une technique analogue, estimaient à 93 heures le temps nécessaire à la totalité d'une dose de ${ }^{45} \mathrm{Ca}$ ingérée pour parcourir tout le tube digestif de veaux de 6 mois. Bien entendu, ces résultats ne peuvent fournir une idée précise sur la durée de transit du repas total, puisque le ${ }^{32} \mathrm{P}$ incorporé ne marque pas intimement le lait et que la faible fraction non absorbée suit probablement la phase liquide du contenu digestif.

\section{$\left.4^{\circ}\right)$ Conclusions sur le besoin d'entretien en phosphore}

L'excrétion fécale de $P$ endogène ne constitue pas chez les veaux la totalité des pertes métaboliques (puisque 1'excrétion urinaire est importante) et ne peut donc être assimilée au besoin d'entretien. Toutefois, les apports alimentaires de $\mathrm{P}$ nécessaires pour couvrir les pertes endogènes fécales sont 16 fois plus importantes chez les veaux du régime $\mathrm{LCF}$; ceci prouve que, d'une part, le besoin d'entretien en $\mathrm{P}$ est susceptible de varier considérablement suivant la nature du régime alimentaire, d'autre part, ce besoin peut être aussi important chez le jeune que chez l'adulte : $3,2 \mathrm{~g}$ de $\mathrm{P}$ par jour pour Ioo $\mathrm{kg}$ de poids vif étant 1 'apport minimum nécessaire pour couvrir les besoins d'entretien. Cependant, ces données ne sont valables que si l'on admet la définition classique du besoin d'entretien, définition discutable lorsque le déterminisme de l'excrétion endogène n'est pas établi. En effet, si l'excrétion fécale endogène est un phénomène secondaire permettant l'élimination normale de l'excès de $\mathrm{P}$ absorbé, la totalité du phosphore fécal endogène ne peut être considérée comme une perte métabolique (résultant de l'utilisation des aliments, du renouvellement de certains tissus, etc.), puisque cette perte serait variable suivant l'apport alimentaire et le degré de rétention du phosphore.

$$
\text { Reçu pour publication en mai } 19^{6} 3 \text {. }
$$

\section{SUMMARY}

THE INFLUENCE OF THE NATURE OF DIET ON THE ENDOGENOUS FAECAL LOSS OF PHOSPHORUS BY THE CALF

The proportion of endogenous phosphorus in the total faecal phosphorus has been determined in six calves aged from seven to eight weeks, receiving two different diets : three calves were given only whole milk (diet L) and three calves were given a diet of early weaning composed of a dry concentrated feed and hay (diet LCF). 
In each group the isotope dilution method, after an injection of ${ }^{32} \mathrm{P}$, was used for two calves. and the study of the variations of the faecal and urinary excretions of an ingested dose of radioisotope was effected on one calf.

The proportion of endogenous $\mathrm{P}$ in the total faecal $\mathrm{P}$ was $5^{\mathrm{I}} \mathrm{p}$. 1oo in the calves with $\operatorname{diet} \mathrm{L}$ and 45 p. Ioo in the calves of diet LCF ; but, because of a faecal excretion of total phosphorus, higher in the calves receiving concentrated feed and hay, the daily endogenous losses of $P$ amount to $1,9 \mathrm{~g}$ for the diet LCF and to o, $15 \mathrm{~g}$ only for the diet L, i. e. 13 times less (tabl. 4).

Several hypotheses have been formulated to explain these differences between diets and to discuss the determinism of the faecal excretion of endogenous $P$.

The true availability of $\mathrm{P}$ was $98 \mathrm{p}$. I oo for milk and $80 \mathrm{p}$. 100 for dry feeds. The physical nature of milk, which does not favour the metabolic losses of mineral elements in the faeces, seems to be an important factor in the very good apparent availability of $\mathrm{P}$ in milk (97 p. 100).

The study of the graphs representing the evolution of the accumulated excretions of ${ }^{32} \mathrm{P}$ in the urine and the faeces (fig. $3,4,5$ and 6) after ingestion of a labelled meal, is particularly interesting. Thus, at the end of eight days, $6 \mathrm{p}$. 100 of the ingested dose of ${ }^{22} \mathrm{P}$ was eliminated in the excreta by the calves with diet LCF as against only I4 p. I00 in the calves receiving only milk. Moreover, the transit time in the gastro-intestinal tract of the whole of the $\mathrm{P}$ of a milk-meal has been estimated as about ninety hours.

These experiments show in particular that the maintenance requirement in $\mathrm{P}$ may be very high in young animals and that this requirement may vary considerably with the nature of the diet.

\section{RÉFÉRENCESS BIBLIOGRAPHIQUES}

Gueguen L., 1962. L'utilisation digestive réelle du phosphore du foin de Luzerne par le Mouton, mesurée à l'aide de ${ }^{32} \mathrm{P}$. Ann. Biol. anim. Bioch. Biophys., 2, 143-149.

Gueguen L., Mathieu C-M., 1962. L'utilisation des éléments minéraux de la ration par le Veau. I. Infuence du régime alimentaire. Ann. Zootech., 11, i I $5^{-1} 34$.

Hansard S. L., Comar C. L., Davis G. K., i 954 . Effects of age upon the physiological behavior of calcium in cattle. Am. J. Physiol., 177, 383-389.

Kleiber M., Smith A. H., Ralsten N. P., Black A. L., I95I. Radio-phosphorus ( ${ }^{32}$ P) as tracer for measuring endogenous phosphorus in cow's feces. J. Nutr., 70, 58-62.

LOFGREeN G. P., KLEIBER M., 1954. Further studies on the availability of phosphorus in alfalfa hay. $J$. Anim. Sci., 13, 258-264.

SMITH R. H., 196r. Effect of the ingestion of wood shavings on magnesium and calcium utilization by milk-fed calves. J. Agric. Sci., 56, 343-350. 